\title{
PRIME IDEALS IN A LARGE CLASS OF NONASSOCIATIVE RINGS
}

\author{
BY \\ PAUL J. ZWIER( $\left.{ }^{1}\right)$
}

\begin{abstract}
In this paper a definition is given for a prime ideal in an arbitrary nonassociative ring $N$ under the single restriction that for a given positive integer $s \geqq 2$, if $A$ is an ideal in $N$, then $A^{s}$ is also an ideal. ( $N$ is called an $s$-naring.) This definition is used in two ways. First it is used to define the prime radical of $N$ and the usual theorems ensue. Second, under the assumption that the $s$-naring $N$ has a certain property $(\alpha)$, the Levitzki radical $L(N)$ of $N$ is defined and it is proved that $L(N)$ is the intersection of those prime ideals $P$ in $N$ whose quotient rings are Levitzki semisimple. $N$ has property $(\alpha)$ if and only if for each finitely generated subring $A$ and each positive integer $m$, there is an integer $f(m)$ such that $A^{f(m)} \subseteq A_{m}$. (Here $A_{1}=A^{s}$ and $A_{m+1}=A_{m}^{s}$.)

Furthermore, conditions are given on the identities an $s$-naring $N$ satisfies which will insure that $N$ satisfies $(\alpha)$. It is then shown that alternative rings, Jordan rings, and standard rings satisfy these conditions.
\end{abstract}

1. Introduction. Several definitions have been proposed for the notion of prime ideal in arbitrary nonassociative rings (narings). Already in [2] S. A. Amitsur suggested a definition of prime ideal in abstract algebras which need not be rings or semigroups. He assumes that the algebra possesses a function $f\left(x_{1}, x_{2}, \ldots, x_{n}\right)$ which has the property that $f\left(x_{1}, x_{2}, \ldots, x_{n}\right)=0$ if one of the $x_{1}=0$. Thus, Amitsur proposed that the ideal $P$ (the kernel of a homomorphism) is prime if

$$
f\left(P_{1}, P_{2}, \ldots, P_{n}\right)=\left\{f\left(p_{1}, p_{2}, \ldots, p_{n}\right): p_{i} \in P_{i}\right\} \subseteq P
$$

where the $P_{i}$ are ideals implies that at least one $P_{i} \subseteq P$.

In [3], Brown and McCoy give a definition of a $u$-prime ideal where $u\left(x_{1}, x_{2}, \ldots, x_{n}\right)$ is a given product of the indeterminates $x_{i}$ in some association. Recently Tsai [8] has given a definition for Jordan rings which uses the operator $b U_{a}=2 a(a b)-a^{2} b$. Examination of the Brown-McCoy paper reveals that a more

Presented to the Society, April 14, 1969 under the titles Prime ideals and the prime radical in a class of narings and The Levitzki radical in a class of narings; received by the editors June 6, 1970.

AMS 1969 subject classifications. Primary 1710.

Key words and phrases. s-naring, prime ideal, semiprime ideal, prime radical, Levitzki radical, $G$-system, $G^{\prime}$-system, $G$-radical, $G^{\prime}$-radical, alternative ring, standard ring, generalized standard ring.

(1) This research was done during the period September 1968 through August 1969 while the author was a National Science Foundation Faculty Fellow in residence at Stanford University. 
general definition of prime ideal could also be given in terms of sums of such $u$ 's. Thus, the Tsai paper actually uses $u\left(x_{1}, x_{2}\right)=x_{1}\left(x_{1} x_{2}\right)+x_{1}\left(x_{1} x_{2}\right)-\left(x_{1} x_{1}\right) x_{2}$. Furthermore, crucial to Tsai's arguments is the fact that for each ideal $U$ in a Jordan ring, $U^{3}$ is also an ideal.

In this paper we introduce the notion of an s-naring; namely a ring in which the sth power of an ideal in the ring is also an ideal in the ring. Using Amitsur's suggestion and a particular $f$, we give a definition for a prime ideal in an $s$-naring. Thus we are able to give a definition of prime radical and semiprime radical for any ideal and hence Tsai's results are generalized to the class of $s$-narings.

Tsai [9] has used his definition of prime ideal to define the Levitzki radical of a Jordan ring; he also gives a characterization of this radical as the intersection of a certain class of prime ideals. Here we define the Levitzki radical for a certain class of $s$-narings, and we too give a characterization of this radical as the intersection of a class of prime ideals. It is an immediate consequence of this characterization that the prime radical for this class of $s$-narings is contained in its Levitzki radical.

2. Preliminaries. The basic definitions and elementary facts concerning narings are contained in [5]. We shall need the following definition.

Definition 2.1. Let $N$ be an arbitrary naring. If $A_{1}, A_{2}, \ldots, A_{k}$ are ideals in $N$, we define $A_{1} A_{2} \cdots A_{k}$ to be the set of all finite sums of terms of the form $a_{1} a_{2} \cdots a_{k}$ where $a_{i} \in A_{i}$ and the elements may be associated in any possible way in the product. We shall denote by $A^{k}$ the set $A A \cdots A$ ( $k$ times). Clearly $A^{k+1} \subseteq A^{k}$ for every positive integer $k$.

Given an arbitrary naring, it may be that for a given ideal $A, A^{k}$ is not an ideal for some integer $k \geqq 2$. In fact, as the following example shows, there are narings having an ideal $A$ such that no power of it (greater than 1) is an ideal.

EXAMPLE 2.1. Let $N$ be the commutative ring generated by $x_{0}, x_{1}, \ldots, x_{n}, \ldots$ where $x_{0}$ is the identity and $x_{1} x_{2}=x_{3}, x_{1} x_{3}=x_{2}, x_{1} x_{4}=x_{3}, x_{1} x_{5}=x_{4}, \ldots, x_{1} x_{n+1}$ $=x_{n}, \ldots$, and $x_{2}^{2}=x_{3}, x_{3}^{2}=x_{4}, \ldots, x_{n}^{2}=x_{n+1}, \ldots$, and all other products are 0 . If we let $A$ be the subring generated by $x_{2}, x_{3}, \ldots, x_{n}, \ldots$, that is,

$$
A=\left[x_{2}, x_{3}, \ldots, x_{n}, \ldots\right]
$$

then $A$ is an ideal, and

$$
\begin{aligned}
A^{2} & =\left[x_{3}, x_{4}, \ldots, x_{n}, \ldots\right] \\
A^{3} & =\left[x_{4}, x_{5}, \ldots, x_{n}, \ldots\right]
\end{aligned}
$$

and in general,

$$
A^{k}=\left[x_{k+1}, x_{k+2}, \ldots, x_{n}, \ldots\right] .
$$

Furthermore, while $A$ is an ideal, $A^{k}$ is not an ideal for any $k \geqq 2$. Notice that $A^{k} \neq A^{j}$ for distinct $k$ and $j$.

Definition 2.2. Let $s$ be an integer greater than 1. The naring $N$ is an $s$-naring if $N$ has the property that if $A$ is any ideal in $N$, then $A^{s}$ is also an ideal in $N$. We 
shall assume that the integer $s$ is fixed throughout our discussions and that for a given naring, $s$ is the minimal such integer.

An associative ring is obviously a 2-ring. The fact that a Lie ring is a 2-ring follows from the Jacobi identity $(a b) x=-(b a) x-(x a) b$ and from the fact that $x y=-y x$. The fact that an alternative ring is a 2-ring can be seen by observing that if $a$ and $b$ are elements of an ideal $A$ and if $x$ is an arbitrary element in the alternative ring, then $(a b) x=(a, b, x)+a(b x)$, where $(a, b, x)=(a b) x-a(b x)$. But in an alternative ring, $(a, b, x)$ is alternating. Thus, $(a, b, x)=-(a, x, b)$. Hence, $(a b) x=-(a x) b+a(x b)+a(b x)$, and each term is in $A^{2}$. A similar argument shows that $x(a b) \in A^{2}$.

It is well known that Jordan rings may have ideals whose square is not an ideal. Yet Jordan rings are 3-rings. In fact, Thedy [10] has recently given conditions on the identities that a naring satisfies which insure that it is a 3-naring. Thus he has shown that the generalized standard rings as introduced by R. D. Schafer [6] are 3-narings. This shows that Jordan rings and also standard rings (introduced by Albert [1]) are 3-narings.

Definition 2.3. Let $P$ be an ideal in the $s$-naring $N . P$ is a prime ideal in $N$ if $P$ has the property that if $A_{1}, A_{2}, \ldots, A_{s}$ are any ideals in $N$ and if $A_{1} A_{2} \cdots A_{s} \subseteq P$, then $A_{1} \subseteq P$, or $A_{2} \subseteq P, \ldots$, or $A_{s} \subseteq P$.

Following Brown and McCoy in [3], we make the following definitions.

Definition 2.4. A nonempty subset $M$ of an $s$-naring $N$ is a $G$-system if whenever $A_{1}, A_{2}, \ldots, A_{s}$ are ideals in $N$ such that $A_{1} \cap M \neq \varnothing, A_{2} \cap M \neq \varnothing, \ldots$, and $A_{s} \cap M \neq \varnothing$, then $A_{1} A_{2} \cdots A_{s} \cap M \neq \varnothing$.

Definition 2.5. Let $A$ be an ideal in the $s$-naring $N$. The $G$-radical of $A$, denoted $A^{G}$, is $\{n \in N$ : any $G$-system which contains $n$ meets $A\}$.

Definition 2.6. An ideal $P$ in an $s$-naring $N$ is a semiprime ideal if for any ideal $A$ in $N$ with $A^{s} \subseteq P$, it follows that $A \subseteq P$.

Definition 2.7. A nonempty subset $M$ of an $s$-naring $N$ is a $G^{\prime}$-system if for any ideal $A$ of $N$ with $A \cap M \neq \varnothing$, it follows that $A^{s} \cap M \neq \varnothing$.

Definition 2.8. Let $A$ be an ideal in the $s$-naring $N$. The $G^{\prime}$-radical of $A$, denoted $A_{G}$, is $\left\{n \in N\right.$ : any $G^{\prime}$-system which contains $n$ meets $\left.A\right\}$.

To illustrate these definitions, consider the following naring as defined by Brown and $\mathrm{McCoy}$ in [3].

EXAMPLE 2.2. Let $R$ be the commutative ring over $I / 2$ with basis $z_{0}, z_{1}, z_{2}, z_{3}$, $\ldots, z_{n}$ with $n>3$. Define multiplication on $R$ as follows:

$z_{0}$ is the multiplicative identity;

$$
z_{1} z_{2}=z_{3} . \quad z_{1} z_{n}=z_{3}, \quad z_{3}^{2}=z_{4}, \quad z_{4}^{2}=z_{5}, \ldots, z_{n-1}^{2}=z_{n}, \quad z_{n}^{2}=z_{2} ;
$$

all other products are 0.

It is shown in [3] that the linear spaces $M=\left[z_{1}, z_{2}, z_{3}, \ldots, z_{n}\right]$ and $N=\left[z_{2}, z_{3}, \ldots, z_{n}\right]$ are the only proper ideals in $R$. Furthermore, calculations will 
show that $M^{2}=N$ and hence that $M^{2}$ is an ideal in $R$. But,

$$
N^{2}=\left[z_{2}, z_{4}, z_{5}, \ldots, z_{n}\right]
$$

while

$$
N^{3}=N^{4}=\left[z_{2}, z_{5}, \ldots, z_{n}\right]
$$

and

$$
N^{5}=N^{6}=N^{7}=N^{8}=\left[z_{2}, z_{6}, \ldots, z_{n}\right],
$$

and continuing, one finds that

$$
N^{2^{n-3+1}}=\left[z_{2}\right] \text { and } N^{2^{n-2}+1}=(0) .
$$

Notice that $N^{k}$ is not an ideal for $2 \leqq k \leqq 2^{n-2}$, and hence, $R$ is a $\left(2^{n-2}+1\right)$-naring. Clearly $M$ is a prime ideal while $N$ and (0) are not. It will be clear later that $M^{G}=M_{G}=M, N^{G}=N_{G}=M$, and that $(0)^{G}=(0)_{G}=M$.

3. Characterization of the $G$ - and $G^{\prime}$-radical. We shall show that, as is the case with associative rings and also with the $Q$-radical defined by Tsai for Jordan rings, the prime radical $A^{G}$ and the semiprime radical $A_{G}$ are the same for any ideal $A$ in the $s$-naring $N$. We shall also show that $A^{G}$ is the intersection of all the prime ideals containing $A$ and also that $A_{G}$ is the intersection of all the semiprime ideals containing $A$. The following lemma is easy to prove.

Lemma 3.1. Let $P$ be an ideal in the s-naring $N$. The following are equivalent:

(1) If $A_{1}, A_{2}, \ldots, A_{s}$ are ideals in $N$ and $A_{1} A_{2} \cdots A_{s} \subseteq P$, then $A_{1} \subseteq P$, or $A_{2} \subseteq P, \ldots$, or $A_{s} \subseteq P$.

(2) If $A_{1}, A_{2}, \ldots, A_{s}$ are ideals in $N$ and $A_{1} \cap \bar{P} \neq \varnothing, A_{2} \cap \bar{P} \neq \varnothing, \ldots$, and $A_{s} \cap \bar{P} \neq \varnothing$, then $A_{1} A_{2} \cdots A_{s} \cap \bar{P} \neq \varnothing$. (Here, $\bar{P}$ is the set-theoretic complement of $P$.)

(3) If $a_{1}, a_{2}, \ldots, a_{s}$ are elements in $\bar{P}$, then $\left[a_{1}\right]\left[a_{2}\right] \cdots\left[a_{s}\right] \cap \bar{P} \neq \varnothing$, where $\left[a_{i}\right]$ is the ideal generated by $a_{i}$.

Proof. That (1) is equivalent to (2) is trivial, as is the implication of (3) by (2). To show that (2) follows from (3), suppose $A_{1}, A_{2}, \ldots, A_{s}$ are ideals in $N$ with $A_{1} \cap \bar{P} \neq \varnothing, A_{2} \cap \bar{P} \neq \varnothing, \ldots, A_{s} \cap \bar{P} \neq \varnothing$. Then there are elements $a_{1}$ in $A_{1} \cap \bar{P}$, $a_{2}$ in $A_{2} \cap \bar{P}, \ldots$, and $a_{s}$ in $A_{s} \cap \bar{P}$. But then by (3), $\left[a_{1}\right]\left[a_{2}\right] \cdots\left[a_{s}\right] \cap \bar{P} \neq \varnothing$. So we have $A_{1} A_{2} \cdots A_{s} \cap \bar{P} \supseteq\left[a_{1}\right]\left[a_{2}\right] \cdots\left[a_{s}\right] \cap \bar{P} \neq \varnothing$, and hence, $A_{1} A_{2} \cdots A_{s} \cap \bar{P} \neq \varnothing$.

THEOREM 3.2. If $A$ is an ideal in the s-naring $N$, then $A^{G}$ is the intersection of all the prime ideals $P$ which contain $A$.

Proof. Let $b \in A^{G}$, and suppose $P$ is a prime ideal containing $A$. It follows from the preceding lemma that $\bar{P}$ is a $G$-system not meeting $A$ so that by definition of $A^{G}$ we must have $b \in P$. Thus $A^{G}$ is contained in every prime ideal $P$ containing $A$ and hence is contained in their intersection. 
Conversely, suppose $b \notin A^{G}$. Then there is a $G$-system $M$ containing $b$ such that $M \cap A=\varnothing$. Apply Zorn's lemma to get a maximal ideal $P$ containing $A$ such that $P \cap M=\varnothing$. We prove that $P$ is a prime ideal in $N$. Suppose $A_{1}, A_{2}, \ldots, A_{s}$ are ideals in $N$ such that $A_{i} \cap \bar{P} \neq \varnothing$ for all such $i$. Since $P$ is a maximal ideal containing $A$ which does not meet $M$, the ideals $P+A_{1}, P+A_{2}, \ldots, P+A_{s}$, being ideals containing $A$, all meet $M$. Hence $\left(A_{1}+P\right)\left(A_{2}+P\right) \cdots\left(A_{s}+P\right)$ meets $M$. But if $\left(a_{1}+p_{1}\right)$ $\left(a_{2}+p_{2}\right) \cdots\left(a_{s}+p_{s}\right)$ is an element of $\left(A_{1}+P\right)\left(A_{2}+P\right) \cdots\left(A_{s}+P\right)$, where the elements are multiplied in some association, then $\left(a_{1}+p_{1}\right)\left(a_{2}+p_{2}\right) \cdots\left(a_{s}+p_{s}\right)$ $=a_{1} a_{2} \cdots a_{s}+$ the sum of terms of the form $a_{1} a_{2} \cdots p_{t} \cdots p_{q} \cdots a_{s}$ where the terms are associated in the same associated way. But each of these terms except the first are clearly in $P$. Hence $\left(A_{1}+P\right)\left(A_{2}+P\right) \cdots\left(A_{s}+P\right) \subseteq A_{1} A_{2} \cdots A_{s}+P$, and thus the latter meets $M$. Therefore $P$ is prime, and $b \notin P$.

A similar theorem can be proved for the $G^{\prime}$-radical. We shall state the theorem, but we shall not give a proof since it is very similar to that of Theorem 3.2.

THEOREM 3.3. If $A$ is an ideal in the s-naring $N$, then $A_{G}$ is the intersection of all the semiprime ideals which contain $A$. Furthermore, $A_{G}$ is a semiprime ideal and an ideal is a semiprime ideal if and only if $A=A_{G}$.

LemMA 3.4. Let a be an element of an s-naring $N$, and let $T$ be a $G^{\prime}$-system containing $a$. Then there is $a$ G-system containing a and contained in $T$.

Proof. Define the sequence $M$ as follows. Let $a_{1}=a$, and whenever $a_{k}$ is defined, define $a_{k+1}$ to be an element of $\left[a_{k}\right]^{s} \cap T$. (Since $a_{k} \in T,\left[a_{k}\right]^{s} \cap T \neq \varnothing$.) We show that $M=\left\{a_{k}\right\}$ is a $G$-system. $M$ is clearly contained in $T$.

Let $A_{1}, A_{2}, \ldots, A_{s}$ be ideals meeting $M$. Choose elements $a_{i_{1}}, a_{i_{2}}, \ldots, a_{i_{s}}$ where $a_{i_{j}}$ comes from $A_{j}$ and each $a_{i_{j}} \in M \subseteq T$. Then, since each of $\left[a_{i_{1}}\right],\left[a_{i_{2}}\right], \ldots,\left[a_{i_{s}}\right]$ meets $T,\left[a_{i_{1}}\right]\left[a_{i_{2}}\right] \cdots\left[a_{i_{s}}\right]$ meets $T$. Now $a_{k+1} \in\left[a_{k}\right]^{s}$, and thus $\left[a_{k+1}\right] \subseteq\left[a_{k}\right]$ for each positive integer $k$. It follows that $\left[a_{j}\right] \subseteq\left[a_{k}\right]$ for $j>k$. Let $t$ be the largest of $i_{1}$, $i_{2}, \ldots, i_{s}$. Then $a_{t+1} \in\left[a_{t}\right]^{s} \subseteq\left[a_{i_{1}}\right]\left[a_{i_{2}}\right] \cdots\left[a_{i_{s}}\right] \cap M$ and hence $a_{t+1} \in A_{1} A_{2} \cdots A_{s} \cap M$.

THEOREM 3.5. If $A$ is any ideal in the s-naring $N$, then $A^{G}=A_{G}$; that is, the $G$-radical and the $G^{\prime}$-radical of $A$ are the same.

Proof. It is clear from the definitions that every prime ideal in $N$ is also a semiprime ideal. Thus $A^{G} \supseteq A_{G}$. Conversely, if $x \in A^{G}$ and $T$ is a $G^{\prime}$-system containing $x$, then there is a $G$-system $M$ such that $x \in M \subseteq T$. Since $x \in A^{G}, M$ meets $A$ and thus $T$ meets $A$. We have shown, therefore, that $A^{G} \subseteq A_{G}$, and hence the two sets are equal.

We shall call the set $A^{G}$ the prime radical of the ideal $A$. The prime radical of the ideal (0) will be called the prime radical of the s-naring $N$. It will be denoted by $R(N)$. Our characterization shows that $R(N)$ is the intersection of all the prime ideals in $N$.

THEOREM 3.6. Let $N$ be an s-naring and $R(N)$ the prime radical of $N$. Then $R(N / R(N))=(0)$. 
Proof. Notice that although $N / R(N)$ is an $s$-naring, the integer $s$ may not be the minimal such in this case. Nevertheless, we may still form the prime radical of $N / R(N)$ relative to $s$. Let $p: N \rightarrow N / R(N)$ be the natural homomorphism. Clearly the image of a prime ideal in $N$ is also a prime ideal in $N / R(N)$. Let $\bar{a} \in R(N / R(N))$, and let $P$ be any prime ideal in $N$. Then $\bar{P}=p(P)$ is a prime ideal in $N / R(N)$. Thus $\bar{a} \in \bar{P}$, and hence $a \in P$ for each prime ideal $P$. This means that $a \in R(N)$ and $\bar{a}=0$.

If $a$ is an element in the $s$-naring $N$, there are various ways to define the powers of $a$. We shall specify one such. Define $a^{1}=a$, and whenever $a^{k}$ is defined, define $a^{k+1}=a \cdot a^{k}$. We shall call an ideal in $N$ a nilideal if for each element $a$ in the ideal there is an integer $k$ such that $a^{k}=0$.

Lemma 3.7. Let $A$ be an ideal in the s-naring $N$ and let $a \in A_{G}$. Then there is $a$ positive integer $k$ such that $a^{k} \in A$.

Proof. Let $M=\left\{a, a^{s}, a^{s^{2}}, a^{s^{3}}, \ldots, a^{s^{k}}, \ldots\right\}$. It is clear that $M$ is a $G^{\prime}$-system in $N$, since if $C$ is an ideal in $N$ and if $a^{s j} \in M \cap C$, then $a^{s+1} \in C^{s} \cap M$. Therefore, the $G^{\prime}$-system $M$ meets $A$, and hence there is a positive integer $k$ such that $a^{k} \in A$.

Notice that if $a$ is an element of the prime radical of $N$, then $a \in(0)_{G}$ and thus there is a positive integer $k$ such that $a^{k} \in(0)$, or $a^{k}=0$. Thus the prime radical of $N$ is a nilideal.

THEOREM 3.8. The prime radical of an s-naring $N$ is a nilideal.

An ideal $A$ in $N$ is a nilpotent ideal if there is a positive integer $k$ such that $A^{k}=(0)$. Also, following the usual custom, we shall call an $s$-naring $N G$-semisimple if $R(N)=(0)$.

THEOREM 3.9. An s-naring $N$ is $G$-semisimple if and only if $N$ contains no nonzero nilpotent ideal.

Proof. Clearly $N$ is $G$-semisimple iff ( 0$)$ is a semiprime ideal. Suppose $N$ contains a nonzero nilpotent ideal $A$ and suppose $k$ is an integer $\geqq 2$ such that $A^{k}=(0)$. Then there is a positive integer $d$ such that $A^{s^{d}}=(0)$, but $A^{s^{d-1}} \neq(0)$. This means that (0) is not semiprime. Notice that $A^{s^{d-1}}$ is an ideal in $N$.

Conversely, if $N$ contains no nonzero nilpotent ideals and (0) is not semiprime, there is an ideal $A$ in $N$ such that $A^{s}=(0)$ but $A \neq(0)$. This is impossible.

COROLlaRY 3.10. The prime radical of an s-naring contains all the nilpotent ideals.

Proof. If $C$ is a nilpotent ideal in $N$, let $\bar{C}$ be the image of $C$ under the natural homomorphism from $N$ to $N / R(N)$. Suppose $C$ is not contained in $R(N)$. Then $\bar{C}$ is a nonzero ideal in $N / R(N)$, and $\bar{C}$ is a nilpotent ideal. This is impossible since $N / R(N)$ is prime semisimple.

Since a naring is isomorphic to a subdirect sum of narings $A_{i}$ if and only if it contains a class of ideals $\left\{B_{i}\right\}$ such that $\bigcap B_{i}=(0)$ and $N / B_{i} \simeq A_{i}$, the following theorem is immediate. 
THEOREM 3.11. A necessary and sufficient condition for an s-naring $N$ to be isomorphic to a subdirect sum of prime subrings is that the naring be prime semisimple.

Without a chain condition on ideals, it is well known that a ring may not contain a maximal nilpotent ideal. One might ask the question whether an $s$-naring having a maximal nilpotent ideal $T$ necessarily must have $T$ equal to its prime radical. The answer is that it need not as Example 2.2 shows. Here the prime radical of $R$ is the ideal $M$ and yet the ideal $N$ is a maximal nilpotent ideal in $R$ which is properly contained in $M$.

In a written communication Michael Rich has pointed out to me that if the Baer lower radical $B(N)$ of an $s$-naring $N$ is defined precisely, as is done for an associative ring, two theorems follow.

THEOREM 3.12. In an s-naring $N$, the Baer lower radical is the intersection of all ideals $Q$ of $N$ such that $N / Q$ has no nonzero nilpotent ideals.

THEOREM 3.13. In any s-naring $N$, the Baer lower radical $B(N)$ is precisely the same as the prime radical $R(N)$.

The proof for Theorem 3.12 is the same as that for associative rings. The proof for Theorem 3.13 is essentially that given by Amitsur in [2] on p. 127.

4. The Levitzki radical for a class of $s$-narings. Recently Tsai [8] has given a definition for the Levitzki radical of a Jordan ring. In doing so he uses the prime ideals as he defined them and then he characterizes the Levitzki radical of these Jordan rings as the intersection of those prime ideals whose quotient rings are Levitzki semisimple. In this section we give a definition for the Levitzki radical for a more general class of $s$-narings and also we shall prove the corresponding characterization for this case.

Definition 4.1. Inductively define the following sets in the $s$-naring $N$. If $A$ is an ideal in $N, A_{1}=A^{s}$, and whenever $A_{m}$ is defined, define $A_{m+1}=A_{m}^{s}$. In the usual way we also define $A_{(0)}=A$, and whenever $A_{(m)}$ is defined, $A_{(m+1)}=A_{(m)} A_{(m)}$. It is easy to check that for each positive integer $m, A_{m} \subseteq A_{(m)}$. Notice that $A_{m}$ is an ideal in $N$ for each positive integer $m$.

In order to define the Levitzki radical of an s-naring we shall make further restrictions on the class of $s$-narings considered. We shall consider only those $s$-narings with the property $(\alpha)$; that is, if $A$ is any subring which is generated by $n$ elements, then there is a positive integer $f(m, n)$ such that $A^{f(m, n)} \subseteq A_{m}$ for each positive integer $m$. Of course the associative rings satisfy the property $(\alpha)$; recently Ževlakov [11] has proved that the same holds for Jordan rings. We shall give sufficient conditions that an $s$-naring $N$ will satisfy the condition $(\alpha)$.

The usual definition of the Levitzki radical of a ring involves the concept of local nilpotence; the idea of local solvability is also useful.

DEFINITION 4.2. An s-naring is locally nilpotent (solvable) if each finitely generated subring is nilpotent (solvable). 
The next lemmas will show that an $s$-naring satisfying property $(\alpha)$ possesses a maximal locally nilpotent ideal. We shall also show that such an ideal is also locally solvable. This will lead naturally to the definition of the Levitzki radical of an $s$-naring satisfying property $(\alpha)$. The development parallels that of Ževlakov [11].

Let $N$ be an $s$-naring which is finitely generated, and let $X$ be a set of generators for $N$. Let $S$ be the set of all nonassociative, noncommutative words that can be formed using the elements of $X$. We define a linear order on $X$ as follows: first linearly order the words of $S$ of length 1 ; that is, linearly order the set $X$; next order the set of words of length 2 ; continue and order the sets of words of length 3, 4, and so forth. Agree that a word of length $k$ is less than a word of length $h$ where $k<h$.

For each element $a$ in $S$, one can define two translations of $N$ : if $x \in N, x R_{a}=x a$, $x L_{a}=a x$. We shall use the notation $T_{a}$ to denote either of these translations. Let $Y=\left\{T_{a}: a \in S\right\}$. Furthermore, let $V$ be the set of all associative, noncommutative words that can be formed using the elements of $Y$. Agree to the following order on $Y: T_{a}<T_{b}$ if $a<b$ in $S$; also say that $R_{a}<L_{a}$ for each $a$ in $S$. If $W=T_{a_{1}} T_{a_{2}} \cdots T_{a_{k}}$ is a word in $V$, call $k$ the $N$-length of $W$; let the degree of $W$ be $\sum \operatorname{deg} a_{i}$, where $\operatorname{deg} a_{i}$ is the length of the word $a_{i}$ in $S$.

A word $u$ in $V$ will be called an $X$-normal word for the $s$-naring $N$ if

$$
u=\left(T_{a_{11}} T_{a_{12}} \cdots T_{a_{1 s-1}}\right)\left(T_{a_{21}} T_{a_{22}} \cdots T_{a_{2 s-1}}\right) \cdots\left(T_{a_{k 1}} T_{a_{k 2}} \cdots T_{a_{k s-1}}\right)\left({ }^{2}\right)
$$

where $a_{11}<a_{21}<\cdots<a_{k 1}, a_{12}<a_{22}<\cdots<a_{k 2}, \ldots, a_{1 s-1}<a_{2 s-1}<\cdots<a_{k s-1}$.

We shall now give some conditions that will insure that the $s$-naring $N$ will satisfy property $(\alpha)$. Later we will see that alternative rings, Jordan rings, and standard rings all satisfy these conditions.

(I) $N$ is $r$-reducible (see A. Albert [1]); that is, there is a positive integer $r$ such that if $a$ is a word in $S$ of length $r+1$, then $T_{a}=\sum W_{i}$, where $W_{i}=T_{z_{1}} T_{z_{2}} \cdots T_{z_{k}}$ and $\sum \operatorname{deg} z_{i}=r+1$ and $1<k \leqq r+1$.

(II) The following identities are satisfied in $V$ :

(i) $R_{a} T_{z_{1}} T_{z_{2}} \cdots T_{z_{s-2}} R_{b}=-R_{b} T_{z_{1}} T_{z_{2}} \cdots T_{z_{s-2}} R_{a}+$ words in $V$ using $a, z_{1}, z_{2}, \ldots, z_{s-2}$, and $b$ each of $N$-length less than $s$.

(ii) $L_{a} T_{z_{1}} T_{z_{2}} \cdots T_{z_{s-2}} L_{b}=-L_{b} T_{z_{1}} T_{z_{2}} \cdots T_{z_{s-2}} L_{a}+$ words in $V$ using $a, z_{1}, z_{2}, \ldots, z_{s-2}$, and $b$ each of $N$-length less than $s$.

$$
\begin{aligned}
L_{a} T_{z_{1}} T_{z_{2}} \cdots T_{z_{s-2}} R_{b}= & a_{1} R_{a} T_{z_{1}} T_{z_{2}} \cdots T_{z_{s-2}} R_{b}+a_{2} L_{a} T_{z_{1}} T_{z_{2}} \cdots T_{z_{s-2}} L_{b} \\
& +a_{3} R_{b} T_{z_{1}} T_{z_{2}} \cdots T_{z_{s-2}} L_{a}+\text { words in } V
\end{aligned}
$$

using $a, z_{1}, z_{2}, \ldots, z_{s-2}$, and $b$ each of $N$-length less than $s$. Here $a_{1}, a_{2}$, and $a_{3}$ are -1 , or 0 , or 1 .

(iv) Same as (iii) except that the roles of $L_{a}$ and $R_{b}$ are reversed.

${ }^{2}{ }^{2}$ For some $j, 1<j \leqq s-1$, part of the $k$ th block $T_{a_{k j}} T_{a_{k j+1}} \cdots T_{a_{k s-1}}$ may not appear. 
LEMMA 4.1. Let $N$ be a naring which is $r$-reducible and let $n$ be a positive integer. Then, if $a$ is $a$ word in $S$ of length $r n+1$, then $a=a^{\prime} \sum W_{i}$ where the $N$-length of each $W_{i}$ is no less than $n$.

Proof. Write $a=a^{\prime} T_{w_{1}} T_{w_{2}} \cdots T_{w_{v}}$, where $\sum \operatorname{deg} w_{i}=r n$. If $\operatorname{deg} w_{j}>r$, use property (I) to write it as the sum of products of $T$ 's where each subscript is a word whose degree is at most $r$. Thus $a=a^{\prime} \sum T_{u_{1}} T_{u_{2}} \cdots T_{u_{h}}$ and $\sum \operatorname{deg} u_{i}=r n$. Therefore there must be $n$ or more such $T$ 's in each term.

Using the operations described in (II), it is easy to see that the following statement is true.

LEMMA 4.2. Let $N$ be an s-naring satisfying conditions (I) and (II). Then if $W$ is any word in $V$, then $W$ or $2^{k} W$ for some suitable $k$ can be written as a sum or words in normal form.

THEOREM 4.3. Let $N$ be an s-naring satisfying properties (I) and (II) and which is finitely generated. Then, for each positive integer $m$, there is a positive integer $f(m)$ such that $N^{f(m)} \subseteq N_{m}$.

Proof. The proof is by induction on the integer $m$. If $m=1$, take $f(1)$ to be $s$. Then $N^{s}=N_{1}$. We may therefore assume that the theorem is true for the positive integer $m-1$. Define the numbers $M$ and $K$ as follows: If $p_{k}$ is the number of words in $S$ of length $k$,

$$
M=\sum_{k=1}^{f(m-1)} 2 p_{k} \text { and } K=\sum_{k=1}^{f(m-1)} 2 k p_{k} .
$$

Let $a$ be a word in $S$ of length at least $r[(s-1) K(M+(s-1))+f(m-1)]+1$. Then, by Lemma 4.1, $a=a^{\prime} \sum V_{i}$ where the $N$-length of each of the $V_{i}$ is at least $(s-1) K(M+(s-1))+f(m-1)$. Write $a=a^{\prime} \sum W_{0} W_{1} W_{2} \cdots W_{M+(s-1)}$, where the $N$-length of $W_{0} \geqq f(m-1)$ and the $N$-length of each $W_{i} \geqq K(s-1)$ for $i=1,2, \ldots$, $M+(s-1)$. Now each $W_{i}(i \geqq 1)$ can be written as the sum of terms which are $X$-normal. Thus $W_{i}$ is the sum of terms of the form

$$
\left(T_{z_{11}^{(i)}} T_{z_{12}^{(i)}} \cdots T_{z_{1 s-1}^{(i)}}\right)\left(T_{z_{21}^{(i)}} T_{z_{22}^{(i)}} \cdots T_{z_{2 s-1}^{(i)}}\right) \cdots\left(T_{z_{k i 1}^{(i)}} T_{z_{k i 2}^{(i)}} \cdots T_{z_{k i s-1}^{(i)}}\right)
$$

Now $\sum \operatorname{deg} z_{j 1}^{(i)}+\sum \operatorname{deg} z_{j 2}^{(i)}+\cdots+\sum \operatorname{deg} z_{j s-1}^{(i)} \geqq(s-1) K$. Thus, at least one of these $s-1$ sums is at least $K$. Since $K=\sum_{k=1}^{f(m-1)} 2 k p_{k}$, for some $j_{0}, z_{k_{i} j_{0}}^{(i)}$ has degree at least $f(m-1)$ and thus is an element of $N_{m-1}$. We have shown that in each of these terms there is a translation of the form $T_{a}$ where $a \in N_{m-1}$. If we return to the product $W_{1} W_{2} \cdots W_{M+(s-1)}$ and substitute our expressions for $W_{i}$, we find that in each of the $M+(s-1)$ blocks there is such a term. Thus

$$
a=a^{\prime} W_{0} \sum u_{j} \quad \text { where } u_{j}=Q_{0} T_{b_{1}} Q_{1} T_{b_{2}} Q_{2} \cdots T_{b_{M+(s-1)}} Q_{M+(s-1)}
$$

where $b_{i} \in N_{m-1}$ and $Q_{j}$ are words in $S$ and $Q_{0}$ and $Q_{M+(s-1)}$ or parts thereof may not appear. 
Agree to the following terminology. If $W$ and $V$ are words whose $N$-length is $s-2$,

$$
W=T_{z_{1}} T_{z_{2}} \cdots T_{z_{s-2}} \text { and } \quad V=T_{w_{1}} T_{w_{2}} \cdots T_{w_{s-2}},
$$

$W$ and $V$ are similar if $z_{i} \in N_{m-1}$ if and only if $w_{i} \in N_{m-1}, i=1,2, \ldots, s-2$. Such a $W$ is an $r$-caliber word if there are exactly $r$ such $T_{z_{i}}$ 's such that $z_{i} \in N_{m-1}$.

Notice that if $W=T_{a} T_{z_{1}} T_{z_{2}} \cdots T_{z_{s-2}} T_{b}$, and if $a \in N_{m-1}$ while $b \notin N_{m-1}$, then

$$
W= \pm T_{b} T_{z_{1}} T_{z_{2}} \cdots T_{z_{s-2}} T_{a}+\text { terms of the form } T_{w_{1}} T_{w_{2}} \cdots T_{w_{v}}
$$

where $1 \leqq v \leqq s-2$ and for some $j_{0}, w_{j_{0}} \subseteq N_{m-1}$. This follows from the fact that $N_{m-1}$ is an ideal in $N$.

Returning to (1), by using the operations described in (2), and moving translations of the form $T_{a}$ where $a \in N_{m-1}$ toward $T_{M+(s-2)}$, it is clear that each $u_{j}$ or $2^{k} u_{j}$ may be written as a sum of terms in the following categories:

Type 1. $v=Q_{0} T_{b_{1}} Q_{1} T_{b_{2}} Q_{2} \cdots T_{b_{k-1}} Q_{k-1} T_{b_{k}} Q_{k}$ where $b_{i} \in N_{m-1}$ and the $Q_{j}$ are similar words of caliber 0 and $k \geqq M+(s-1)$.

Type 2. $v=Q_{0} T_{b_{1}} Q_{1} T_{b_{2}} Q_{2} \cdots T_{b_{k-1}} Q_{k-1} T_{b_{k}} Q_{k}$ where $b_{i} \in N_{m-1}$ and the $Q_{j}$ are similar words of caliber 1 and $k \geqq M+(s-2)$.

Type $s-2 . v=Q_{0} T_{b_{1}} Q_{1} T_{b_{2}} Q_{2} \cdots T_{b_{k-1}} Q_{k-1} T_{b_{k}} Q_{k}$ where $b_{i} \in N_{m-1}$ and the $Q_{j}$ are similar words of caliber $s-3$ and $k \geqq M+1$.

Type $s-1$. $v=P T_{b_{1}} T_{b_{2}} \cdots T_{b_{s-1}} Q$ for some words $P$ and $Q, b_{i} \in N_{m-1}$.

If we consider any term of type $q$,

$$
v=Q_{0} T_{b_{1}} Q_{1} T_{b_{2}} Q_{2} \cdots T_{b_{k-1}} Q_{k-1} T_{b_{k}} Q_{k}
$$

where the $Q_{j}$ are similar words of caliber $q-1$ and $k \geqq M+(s-q)$ and if $Q_{k-1}$ $=T_{z_{1}} T_{z_{2}} \cdots T_{z_{s-2}}$, then for some $j_{0}, z_{j_{0}} \notin N_{m-1}$, and since all the $Q$ 's are similar, there are $k$ translations $T_{w_{1}}, T_{w_{2}}, T_{w_{3}}, \ldots, T_{w_{k}}$ such that $w_{j} \notin N_{m-1}$. But $k \geqq M+(s-q) \geqq M+1$, and hence at least two of these words must be the same. Consider two such similar blocks, $Q_{i}$ and $Q_{j}$ :

$$
Q_{i}=T_{z_{1}} T_{z_{2}} \cdots T_{z_{s-2}} \text { and } Q_{j}=T_{w_{1}} T_{w_{2}} \cdots T_{w_{s-2}} .
$$

Assume that $T_{z_{j_{0}}}$ and $T_{w_{j_{0}}}$ have the property that $w_{j_{0}}$ and $z_{j_{0}}$ are not elements of $N_{m-1}$. If these elements are unequal, then we may interchange them and, in addition, we will obtain terms of Type $q+1$ or higher. Thus, we may assume that the equal translations are in adjacent blocks. Again, the block which has these equal translations at the ends collapses giving terms which are of Type $q+1$ or more. Thus it follows that the terms of Type 1 are sums of terms of Type 2 or more; that the terms of Type 2 are sums of terms of Type 3 or more, and so on. Thus $a=a^{\prime} W_{0} \sum V_{i}$ where each word is of Type $s-1$, that is, of the form $P T_{a_{1}} T_{a_{2}} \cdots T_{a_{s-1}} Q$. Hence, having $W_{0}$ of $N$-length at least $f(m-1)$, we have

$$
\begin{aligned}
a & =\sum a^{\prime} W_{0} P T_{a_{1}} T_{a_{2}} \cdots T_{a_{s-1}} Q \subseteq N_{m-1}\left[P T_{a_{1}} T_{a_{2}} \cdots T_{a_{s-1}} Q\right] \\
& \subseteq\left[\cdots\left[\left[\left[N_{m-1}\right] N_{m-1}\right] N_{m-1}\right] \cdots N_{m-1}\right] \subseteq N_{m-1}^{s}=N_{m}
\end{aligned}
$$

and the theorem is proved. 
COROLLARY 4.4. If $N$ is an alternative ring and if $N$ is finitely generated, then for each positive integer $m$ there is a positive integer $f(m)$ such that $N^{f(m)} \subseteq N_{m}$.

Proof. An alternative ring is 1-reducible. This follows from the fact that the following identities are satisfied:

and

$$
R_{x y}=R_{x} R_{y}-L_{x} R_{y}+R_{x} L_{y}
$$

$$
L_{x y}=L_{y} L_{x}+L_{y} R_{x}-R_{x} R_{y}
$$

Since in an alternative ring,

$$
\begin{aligned}
& R_{x} R_{y}=R_{x y}-R_{y} R_{x}+R_{y x} \quad L_{x} R_{x}=R_{x} L_{x} \\
& R_{y} L_{x}=L_{x} L_{y}+L_{x} R_{y}-L_{x y} \quad \text { and } \quad R_{x} R_{x}=R_{x^{2}} \\
& L_{y} R_{x}=R_{x} L_{y}+R_{x} R_{y}-R_{x y} \quad L_{x} L_{x}=L_{x^{2}} \\
& L_{x} L_{y}=-L_{y} L_{x}+L_{x y}+L_{y x}
\end{aligned}
$$

the condition (II) is met. Recall that an alternative ring is a 2-naring.

The fact that Jordan rings satisfy the conditions (I) and (II) follows from the identity

$$
R_{z} R_{x y}-R_{z} R_{y} R_{x}+R_{y} R_{z x}-R_{y(x z)}+R_{x} R_{z y}-R_{x} R_{y} R_{z}=0,
$$

and from the commutativity of multiplication in Jordan rings. Of course, only right translations need be considered in this case.

COROLlaRY 4.5. If $N$ is a standard ring and if $N$ is finitely generated, then for each positive integer $m$, there is a positive integer $f(m)$ such that $N^{f(m)} \subseteq N_{m}$.

Proof. Recall that a standard ring is one in which the following identities are satisfied:

(1) $(x w, y, z)+(x z, y, w)+(w z, y, x)=0$,

(2) $(x, y, z)+(z, x ; y)-(x, z, y)=0$.

If, in (1), we interchange the roles of $x$ and $w$ and then subtract the result from (1), we obtain

(3) $(x w, y, z)=(w x, y, z)$.

Also, if we interchange the roles of $x$ and $z$ in (2) and add, we obtain

(4) $(x, y, z)=-(z, y, x)$.

In terms of translations, identity (1) is written as follows:

(5) $R_{x} R_{y} R_{z}-R_{x} R_{y z}+L_{(x z) y}-L_{y} L_{x z}+R_{z} R_{y} R_{x}-R_{z} R_{y x}=0$.

Thus,

(6) $R_{x} R_{y} R_{z}=-R_{z} R_{y} R_{x}$ mod terms of $N$-length 2 or less, and

(7) $L_{(x z) y}=0$ mod terms of length 2 or more.

Using (3), we obtain the identity

(8) $R_{x} R_{y} R_{z}-R_{x} R_{y z}-L_{x} R_{y} R_{z}+L_{x} L_{y z}=0$

and hence 
(9) $R_{x} R_{y} R_{z}=L_{x} R_{y} R_{z}$ mod terms of length 2 .

Using (4) we obtain $L_{x y}-L_{y} L_{x}+R_{y} R_{x}-R_{y x}=0$. Thus

(10) $L_{y} L_{x}=R_{y} R_{x}$ mod terms of length 1 .

All of this shows that $L_{x} L_{y} L_{z}=L_{x} L_{y} R_{z}=R_{x} R_{y} L_{z}=R_{x} R_{y} R_{z}$ mod terms of length 2 or less. Finally, using (2), we obtain the identity

(11) $R_{y} L_{x}-L_{x} R_{y}=L_{y} L_{x}-R_{x} R_{y}$ mod terms of $N$-length 1 .

Thus, $R_{y} L_{x} R_{z}-L_{x} R_{y} R_{z}=L_{y} L_{x} R_{z}-R_{x} R_{y} R_{z}$ mod terms of $N$-length 2 . But $L_{x} L_{y} R_{z}$ $=R_{x} R_{y} R_{z}$ mod terms of $N$-length 2 or less, and hence $R_{y} L_{x} R_{z}=L_{y} L_{x} R_{z} \bmod$ terms of $N$-length 2 or less. Similarly, $L_{x} R_{y} L_{z}=R_{x} R_{y} L_{z} \bmod$ terms of $N$-length 2 or less. Hence $T_{a} T_{b} T_{c}=-T_{c} T_{b} T_{a}$ mod terms of $N$-length 2 or less and (II) is satisfied.

If we use (4) and (1), we obtain

(12) $(z, y, x w)+(w, y, x z)+(x, z, w z)=0$,

and thus

(13) $R_{y(w x)}=0$ mod terms of $N$-length 2 or more.

Using (2), we find that $R_{y z}=R_{z y} \bmod$ terms of $N$-length 2, and thus $R_{(w x) y}=R_{y(w x)}$ mod terms of $N$-length 2 or more. Similarly, $L_{x z}=L_{z x}$ mod terms of $N$-length 2 and thus $L_{(x y) z}=L_{z(x y)}$ mod terms of $N$-length 2 or more. We have therefore verified that (I) is satisfied. Recall that a standard ring is a 3-naring.

We now return to arbitrary $s$-narings satisfying property $(\alpha)$. It is our purpose to define the Levitzki radical for such a ring. Our theorems parallel those given in the book Rings and radicals by Divinsky [4].

LEMMA 4.6. If $N$ is an s-naring satisfying property ( $\alpha)$, then every finitely generated subring which is solvable is also nilpotent, and conversely.

Proof. Let $A$ be a finitely generated subring and suppose $A$ is generated by $n$ elements. Suppose $A$ is nilpotent. Then there is a positive integer $k$ such that $A^{k}=(0)$. By taking an integer sufficiently large we can find an integer $c$ such that $A_{(c)} \subseteq A^{2^{c}} \subseteq A^{k}=(0)$. Thus $A$ is solvable.

Conversely, if $A$ is solvable, there is a positive integer $m$ such that $A_{(m)}=(0)$. Since $N$ satisfies property $(\alpha)$, there is a positive integer $f(n, m)$ such that $A^{f(n, m)}$ $\subseteq A_{m} \subseteq A_{(m)}$. Clearly $A$ is also nilpotent.

LEMMA 4.7. Let $N$ be an s-naring satisfying property ( $\alpha$ ). If $A$ is a subring of $N$ which is finitely generated, then $A_{m}$ is also finitely generated.

Proof. Suppose $X=\left\{x_{1}, x_{2}, \ldots, x_{n}\right\}$ is a set of generators for $A$ and let $Y$ be the set of all words in $A_{1}=A^{s}$ which are of length less than $f(n, 2)$, where $f(n, 2)$ is that positive integer such that $A^{f(n, 2)} \subseteq A_{2}$. Since $X$ is finite, $Y$ is also finite. We shall show that $Y$ generates $A_{1}$. If $a \in A_{1}$, then $a=\sum_{i} a_{i, 1} a_{i, 2} a_{i, 3} \cdots a_{i, s}$ where $a_{i, j}$ is in $A$ for $j=1,2, \ldots, s$. Now each $a_{i, j}$ is the sum of $X$-words and thus $a=\sum u_{i}$ where the $u_{i}$ are $X$-words in $A_{1}$. If the $X$-length of, say $u_{1}$, is not less than $f(n, 2)$, then $u_{1} \in A_{2}$. Thus $u_{1}=w_{1} w_{2} \cdots w_{s}$ where $w_{i} \in A_{1}$ and the length of $u_{1}$ is $l\left(w_{1}\right)+l\left(w_{2}\right)$ 
$+\cdots+l\left(w_{s}\right)$. Continuing, we can express $a$ as the sum or products of $Y$-words. The proof continues by induction in the obvious way.

THEOREM 4.8. If $N$ is an s-naring satisfying property $(\alpha)$, then $N$ contains a maximal locally nilpotent ideal, the Levitzki radical $L(N)$ of $N$. Furthermore, this maximal locally nilpotent ideal contains all the locally nilpotent ideals in $N$.

Proof. We list the following facts to prove this theorem.

1. There is a locally nilpotent ideal in $N$, namely, (0).

2. If $Q_{1} \subseteq Q_{2} \subseteq Q_{3} \cdots \subseteq Q_{n} \cdots$ is a chain of locally nilpotent ideals in $N$, then $\cup_{i} Q_{i}$ is a locally nilpotent ideal in $N$.

3. From Zorn's lemma, it follows that $N$ has a maximal locally nilpotent ideal $L(N)$.

4. The fact that $L(N)$ contains all the locally nilpotent ideals in $N$ follows from the fact that if $B$ is an ideal in an $s$-naring $A$ satisfying property $(\alpha)$, and if $B$ and $A / B$ are locally nilpotent, then $A$ is also locally nilpotent. This is seen as follows: Let $S$ be a finitely generated subring of $A$, and let $\bar{S}$ be the image of $S$ under the natural homomorphism. Then $\bar{S}$ is finitely generated and since $A / B$ is locally nilpotent and thus locally solvable, $\bar{S}_{(m)}=(0)$ for some $m$. This means that $S_{(m)} \subseteq B$. Now $S_{m}$ is a finitely generated subring of $B$ and since $B$ is locally solvable, $\left[S_{(m)}\right]_{(k)}$ $=(0)$. This means that $S_{(m+k)}=(0)$ and $S$ is solvable and thus nilpotent.

From the above, if $A$ is a locally nilpotent ideal in $N$ which is not contained in $L(N)$, then $(L(N)+A) / A \simeq L(N) / A \cap L(N)$, and since $L(N) / A \cap L(N)$ and $A$ are locally nilpotent, it follows that $A+L(N)$ is locally nilpotent, contradicting the fact that $L(N)$ is maximal.

A proof very similar to that usually given in the associative case can be given for the next theorem. Of course an $s$-naring satisfying property $(\alpha)$ is Levitzki semisimple if and only if its Levitzki radical is (0).

THEOREM 4.9. If $N$ is an s-naring satisfying property $(\alpha)$, then $N / L(N)$ is Levitzki semisimple.

We now characterize $L(N)$ in terms of the prime ideals that we have previously defined.

THEOREM 4.10. The Levitzki radical of an s-naring satisfying property $(\alpha)$ is the intersection of all those prime ideals in $N$ whose quotient rings are Levitzki semisimple.

Proof. Part 1. For each prime ideal $P$ in $N, P \supseteq L(N)$.

For suppose not. Then $L(N)+P$ is an ideal properly containing $L(N)$ and $(L(N)+P) / P \simeq L(N) / P \cap L(N)$ is a nonzero, locally nilpotent ideal in the Levitzki semisimple $s$-naring $N / L(N)$. This is impossible. Thus $L(N) \subseteq \bigcap P=W$.

Part 2. $W \subseteq L(N)$. We shall prove the contrapositive. Suppose $x$ is not in $L(N)$. Then, since $L(N)$ is maximal, $(x)$, the ideal generated by $x$, is not locally nilpotent. Hence there is a finitely generated subring $S$ of $(x)$ generated by say $m$ elements such that $S$ is not nilpotent. Thus, for each positive integer $m, S_{(m)}$ is not nilpotent. 
Notice that $S_{m}$ is finitely generated and if $S_{m}$ were nilpotent, then $S^{f(n, m)} \subseteq S_{m}$ would force $S$ to be nilpotent.

Now let $\sigma$ be the set of all ideals $Q$ in $N$ such that $S_{m} \ddagger Q$, for all $m$. $\sigma$ is nonempty since (0) is in $\sigma$. We now apply Zorn's lemma to get a maximal such ideal. (Let $Q_{1} \subseteq Q_{2} \subseteq Q_{3} \subseteq \cdots \subseteq Q_{n} \subseteq \cdots$ be a chain of ideals each of which has this property. Then $\cup Q_{i}$ is an ideal in $N$. Suppose $S_{m_{0}} \subseteq Q_{i}$ for some $m_{0}$. Then $S_{m_{0}} \subseteq Q_{i_{0}}$ for some $i_{0}$ since $S_{m_{0}}$ is finitely generated. This is a contradiction.) Let $Q$ be a maximal element in $\sigma$. The next project is to show that $Q$ is a prime ideal. Suppose not. Then there are ideals $A_{1}, A_{2}, \ldots, A_{s}$ in $N$ such that $A_{1} A_{2} \cdots A_{s} \subseteq Q$, but $A_{1} \ddagger Q, A_{2} \ddagger Q, \ldots$, and $A_{s} \ddagger Q$. It follows that $A_{1}+Q=A_{1}^{\prime}, A_{2}+Q=A_{2}^{\prime}, \ldots$, $A_{s}+Q=A_{s}^{\prime}$ are ideals properly containing $Q$. Thus, by the maximality of $Q$, there exist positive integers $n_{1}, n_{2}, \ldots, n_{s}$ such that $S_{n_{1}} \subseteq A_{1}^{\prime}, S_{n_{2}} \subseteq A_{2}^{\prime}, \ldots$, and $S_{n_{s}} \subseteq A_{s}^{\prime}$. Taking $t$ to be the maximum of the numbers $n_{1}, n_{2}, \ldots, n_{s}$, we have that $S_{t} \subseteq A_{i}^{\prime}$ for $i=1,2, \ldots, s$. But then $S_{t+1}=S_{t} S_{t} \cdots S_{t}$ ( $s$ times) $\subseteq A_{1}^{\prime} A_{2}^{\prime} \cdots A_{s}^{\prime} \subseteq A_{1} A_{2} \cdots A_{s}+Q$ $=Q$. This contradicts the fact that $S_{m} \ddagger Q$ for all $m$.

Finally we show that $N / Q$ is Levitzki semisimple. Let $\bar{N}=N / Q$ and $\bar{Z}=Z / Q$ where $\bar{Z}$ is a locally nilpotent ideal in $\bar{N}$ which is not zero. Since $Q$ is a prime ideal, $L(N) \subseteq Q$. Thus the subring $S$ that we considered in the previous part of the proof may be taken in $Z$ (recall that $x \notin L(N)$ ). Now $S$ is a finitely generated subring in $Z$ and hence $\bar{S}$ is also finitely generated in $\bar{Z}$. Thus $\bar{S}$ is nilpotent and solvable. Thus there is a positive integer $m$ such that $\bar{S}_{(m)}=(0)$, and hence $\bar{S}_{m} \subseteq \bar{S}_{(m)}=(0)$. From this it follows that $S_{m} \subseteq Q$. This is impossible.

All of the above shows that $x \notin Q$ where $Q$ is a prime ideal which is Levitzki semisimple. Thus $L(N)=\bigcap P$ where $P$ is a prime ideal whose quotient ring is semisimple.

COROLLARY 4.11. Every Levitzki semisimple s-naring satisfying property $(\alpha)$ is isomorphic to a subdirect sum of prime Levitzki semisimple s-narings.

COROLlaRY 4.12. For every s-naring $N$ satisfying $(\alpha)$, the prime radical $R(N)$ is contained in the Levitzki radical $L(N)$.

We close this section with a theorem giving sufficient conditions that the two radicals coincide.

THEOREM 4.13. If $N$ is an s-naring satisfying property $(\alpha)$ and if $N$ satisfies a minimum condition on ideals, then the prime radical $R(N)$ and the Levitzki radical $L(N)$ coincide.

Proof. Since $N$ satisfies the minimum condition on ideals, so does $N / R(N)$. Thus $L(N / R(N))$ being a locally nilpotent ideal is a nilpotent ideal. But $N(R(N))$ is prime semisimple and thus $L(N / R(N))=(0)$. Thus $R(N)$ is a prime ideal and $N / R(N)$ is Levitzki semisimple. So $R(N) \supseteq L(N)$ and thus $R(N)=L(N)$. 


\section{REFERENCES}

1. A. A. Albert, Power-associative rings, Trans. Amer. Math. Soc. 64 (1948), 552-593. MR 10, 349.

2. S. A. Amitsur, A general theory of radicals. II, III, Amer. J. Math. 76 (1954), 100-136. MR 15, 499.

3. B. Brown and N. H. McCoy, Prime ideals in nonassociative rings, Trans. Amer. Math. Soc. 89 (1958), 245-255. MR 20 \#3196.

4. N. J. Divinsky, Rings and radicals, Univ. of Toronto Press, Toronto, 1965.

5. J. Levitzki, Prime ideals and their lower radical, Amer. J. Math. 73 (1951), 25-29. MR 12, 474.

6. R. D. Schafer, Generalized standard algebras, J. Algebra 12 (1969), 386-417.

7. M. Slater, Alternative rings with d.c.c. I, J. Algebra 11 (1969), 102-110. MR 38 \#2184.

8. C. Tsai, The prime radical in Jordan rings, Proc. Amer. Math. Soc. 19 (1968), 1171-1175. MR 37 \#6336.

9. - The Levitzki radical in Jordan rings, Proc. Amer. Math. Soc. 24 (1970), 119-123.

10. A. Thedy, Zum Wedderburnschen Zerlegungssatz, Math. Z. 113 (1970), 175-195.

11. K. A. Ževlakov, Solvability and nilpotence of Jordan rings, Algebra i Logika Sem. 5 (1966), no. 3, 37-58 (Russian). MR 34 \#7601.

\section{Calvin College,}

Grand Rapids, Michigan 49506 